\title{
EVALUACIÓN Y CALIFICACIÓN DEL DESEMPEÑO, DESAFÍOS PARA LA ACTIVIDAD ACADÉMICA: EL CASO DE LA FACULTAD TECNOLÓGICA DE LA USACH
}

\author{
JULIO CÉSAR GONZÁLEZ CANDIA \\ Facultad Tecnológica \\ Universidad de Santiago de Chile \\ Julio.gonzalez@usach.cl
}

\section{RESUMEN}

La teorización y puesta en práctica de procesos de evaluación y calificación han estado vinculadas a la necesidad manifiesta de distintos sectores de la sociedad por la calidad del sistema educativo en sus distintos niveles y ámbitos de acción. La Universidad de Santiago de Chile (USACH) en el marco de procesos de aseguramiento de la calidad, necesidad de apreciar y valorar la contribución de sus docentes y el desarrollo de acciones sistemáticas de una gestión de recursos humanos, en el mes de agosto de 2009 aprobó el Reglamento de Evaluación y Calificación del Desempeño Académico. Esta normativa tiene por objeto evaluary calificar el trabajo realizado por cada uno de sus académicos; constituir un incentivo permanente para el perfeccionamiento de las actividades que sirvan para promovery desarrollar su cuerpo académico y contribuir, en suma, a elevar los niveles de calidad y productividad de la universidad. El presente ensayo junto con la exposición de algunas posiciones teóricas especialmente referidas al concepto de evaluación del desempeño, presenta algunos antecedentes generales de la USACHy de la Facultad Tecnológica y releva los principales desafíos y tensiones del sistema que se ha iniciado en su primera etapa en la ya señalada institución educativa.

PALABRAS CLAVES: EVALUACIÓN, CALIFICACIÓN, DESEMPEÑO ACADÉMICO, FACULTAD TECNOLÓGICA, USACH.

\section{ABSTRACT}

The theoretical and practical process of evaluation and qualification has been directly linked to the concerned expressed by different social sectors in relation to the quality of the educational system in its different levels and areas of action. In august 2009, the University of Santiago of Chile approved, under its framework of quality assurance, the Regulations for Evaluation and Qualification of the Academic Performance, in order to estimate and value the contributions of its professors, and develop systematic actions for human resource management. The aim of this regulation is to evaluate and qualify the job of each of its academicians, to help them grow, to establish a permanent incentive and, in sum, to raise the level in the quality and productivity of the University. The present essay, by presenting some theoretical positions specially referred to the concept of evaluation of the performance, gives some general antecedents of the USACH and of the Technological Faculty and determines the principal challenges and tensions of the system in its first stage in this distinguished educational institution.

KEY WORDS: EVALUATION, QUALIFICATION, ACADEMIC PERFORMANCE, TECHNOLOGICAL FACULTY, USACH. 


\section{INTRODUCCIÓN}

La reflexión, discusión y puesta en práctica de procesos de evaluación y calificación han estado vinculados a la necesidad manifiesta de distintos sectores de la sociedad por la calidad del sistema educativo; y aunque se reconoce que el término de calidad es de carácter pluridimensional, es decir que puede estar referido a aspectos tan distintos como la eficiencia en los procesos, la eficacia en términos de impacto en los resultados de corto, mediano y largo plazo, la relevancia en las tareas emprendidas, la equidad y la transparencia, así como la congruencia y relevancia entre los mismos procesos, los resultados y las demandas sociales; finalmente se termina, por lo general, proponiendo la evaluación - tanto a los actores como a los procesos - como un mecanismo clave para alcanzar la calidad del sector independiente del nivel sobre el cual se concentre el análisis.

Los autores Rueda y Torquemada (2008) indican que:

Comúnmente los puntos de vista de los críticos que señalan los bajos niveles de calidad en la educación superior identifican la labor de los docentes como una de las principales causas de la inconsistencia observada en el aprendizaje de los estudiantes; y con este señalamiento vuelve a aparecer la evaluación, en este caso la referida al desempeño de los docentes, como uno de los medios privilegiados para garantizar la mejora del sistema (p.98).

Cabe mencionar eso sí, que cada vez que se pone en el centro de la discusión primero y la instalación después de sistemas de evaluación docentes, las tensiones y los conflictos entre los actores involucrados no se hacen esperar.

La Universidad de Santiago de Chile (USACH) en el marco de procesos de aseguramiento de la calidad, necesidad de apreciar y medir la calidad y productividad de sus académicos, entre otras razones, en el mes de agosto de 2009 aprueba el Reglamento de Evaluación y Calificación del Desempeño Académico. A esta fecha - mayo de 2012- y junto con haber iniciado un plan piloto con un primer año de precalificación, se enfrenta al desafío de instalar una cultura de evaluación al rendimiento individual en una organización pública, burocrática y tradicional donde nunca antes se había realizado. Durante el año 2011 la comunidad académica se abocó en la discusión y generación de los instrumentos de medición -entiéndase manuales por Facultad- que aseguren la objetividad e imparcialidad del proceso.

\section{METODOLOGÍA}

Para realizar este ensayo se utilizó una metodología preferentemente descriptiva y analítica, que consideró las siguientes etapas:

1) Selección de un tema para elaborar un artículo publicable en la modalidad de ensayo para una revista del área de la administración nacional o -de preferencia- internacional. En la selección del tópico se contó con la valiosa contribución de tres académicas de la Facultad quienes validaron la importancia del tema a tratar tanto a nivel de especialidad o área de la gestión de recursos humanos como para visibilizar su importancia al interior de la misma institución.

2) Una vez seleccionado el tema, en este caso la Evaluación y Calificación del Desempeño Académico en la USACH, se procedió a localizar y sistematizar material teórico de diversos autores para confeccionar un marco de referencia para contextualizar de mejor forma el ensayo. Los criterios de selección de los autores y sus respectivas citas bibliográficas se basaron en dos aspectos: calidad de las fuentes y pertinencia o mayor relación 
de los planteamientos de los autores con el tema a desarrollar.

3) Analizar la resolución exenta que aprobó el Reglamento de Evaluación y calificación del Desempeño Académico en la Universidad de Santiago de Chile.

4) Confección y envío del ensayo a una revista internacional, trabajo que fue concluido a mediados de diciembre del año 2011.

5) Finalmente, con base en las sugerencias del equipo editor de la Revista Nacional de Administración, se procedió a incorporar cada una de las recomendaciones con la finalidad de mejorar el presente trabajo. Esta última tarea concluyó a finales de mayo de 2012.

\section{El concepto de Evaluación del Desempeño}

Una primera pregunta que debiéramos contestar es ¿qué vamos a entender por desempeño en un contexto organizacional? Según Rodríguez (2003):

Se han identificado debilidades en la operacionalización del constructo desempeño, tanto a nivel organizacional como a nivel individual. Estos cuestionamientos parten de que las definiciones típicas de desempeño son deficientes, pues dependen exclusivamente de prescripciones conductuales escritas que sirven de base para centrarse en el examen de los antecedentes situacionales de las conductas pro-sociales como eventos lineales, sin considerar variables como el desempeño contextual y otros valores disposicionales no pre-determinados (p.188).

Una segunda interrogante está relacionada con ¿cuándo y dónde se inició la medición del desempeño? Es importante señalar que las prácticas de Evaluación de Desempeño (ED) no son nuevas, desde que el hombre comenzó a vivenciar el trabajo en conjunto con otras personas, su labor y/o contribución de una u otra forma, directa e indirectamente comenzó a ser evaluada. Fuchs (1997) señala que el uso "formal de un sistema de evaluación del desempeño comenzó a ser utilizado en los gobiernos y en las fuerzas armadas de Estados Unidos en los inicios del siglo pasado en los niveles más bajos de la organización" (p. 2).

Rodríguez (2006, citando a Chiavenato,1998) ha sostenido que durante mucho tiempo los administradores se preocuparon solo por la eficiencia de la máquina como medio para aumentar la productividad, pero esto -obviamente- no logró resolver el problema del aumento de la eficiencia de la organización. Debido a lo anterior, es que se revirtió ese enfoque y la principal necesidad de los administradores pasó a situarse frente al hombre (y la mujer). De ahí que surgieron preguntas del tipo: ¿cómo potenciarlo o desarrollarlo?, ¿qué lo lleva a ser más eficiente y efectivo? y ¿cuál ambiente es el más adecuado para su implementación y funcionamiento?

Sostiene más adelante Rodríguez (2006) que el "factor humano, base de toda acción productiva al generar procesos de trabajo, de pronto olvida revisar su estado de evolución. Eventualmente, los directivos empresariales solicitan cuadros de resultados, no obstante estos se abocan al cumplimiento de metas laborales que son establecidas con base en pronósticos y/o alcances presupuestales" (p. 1). Según los autores Gan y Triginé (2006) la Evaluación de Desempeño es "una apreciación sistemática del valor que una persona demuestra, por sus características personales y/o por sus prestaciones, a la organización de la que forma parte, expresada periódicamente conforme a un preciso procedimiento conducido por una o más personas conocedoras tanto de la persona como del puesto de trabajo" (p. 193). 
Gan y Triginé (2006) han sostenido que la ED "es un procedimiento sistemático idéntico para todos los miembros de la organización -todos son evaluados por sus resultados (objetivos de orientación cuantitativa) y/o por sus actitudes (objetivos cualitativos o competencias), con ello se logra que todos los afectados conozcan los criterios de evaluación y que se establezca una uniformidad de actuación que ayude a interiorizar el sentido de equidad del proceso" (p.193-194).

Otros autores, tales como Dolan, Valle, Jackson y Schuler (2007) hablan de la evaluación del rendimiento. Estos autores sostienen que:

A pesar de que los empleados pueden saber en qué medida están rindiendo adecuadamente a través de medios informales (comentarios de los compañeros de trabajo o de los superiores), la evaluación del rendimiento se define como un procedimiento estructural y sistemático para medir, evaluar e influir sobre los atributos, comportamientos y resultados relacionados con el trabajo, con el fin de descubrir en qué medida es productivo el empleado y si podrá mejorar su rendimiento futuro (p. 229).

El sistema de evaluación del rendimiento engloba un conjunto de factores tales como el análisis del puesto de trabajo, la validez y fiabilidad de los métodos utilizados, las características del evaluador y del sujeto evaluado y el cómo se está utilizando la evaluación del rendimiento en relación a la política y objetivos establecidos a los recursos humanos. Todos estos aspectos pueden afectar a la estructura y procedimiento de medición y evaluación del rendimiento en una organización determinada.

Páez (2001) menciona que: "para llevar a cabo cualquier proceso de evaluación de desempe- ño, es importante y muy pertinente conocer a cabalidad las políticas, las normas y los sistemas de reclutamiento, selección y empleo, así como también los de promoción, de disciplina, de desarrollo y por supuesto de evaluación del desempeño, de compensaciones, beneficios e incentivos no monetarios" (p. 2-3). No solo se trata de conocer a cabalidad, como lo expresa el autor, sino que en la práctica los sistemas de evaluación de desempeño deben estar vinculados de manera efectiva con los otros subsistemas de la gestión de personas. Entre estos podemos destacar el subsistema de entrenamiento y capacitación del personal, el cual tiene un impacto estratégico para las organizaciones. Si las personas resultan mal o deficientemente evaluadas, si se detectan brechas de rendimiento importantes estas deben ser formadas de manera adecuada o nuevamente instruidas con el propósito de superar estas debilidades que redundarán más temprano que tarde en la productividad global de la empresa.

Centrándonos más en el ámbito educativo los autores Catalán y González (2009) en concordancia con otros investigadores han sostenido que "la posibilidad de intervención de los profesores en aspectos que contribuyan al mejoramiento de la calidad de la educación tiene como requerimiento que los propios profesores cambien y que este cambio surja de la evaluación de su quehacer y de lo que acontece en las instituciones en las que lo desarrollan" (p. 4). Esta última es una tarea nada de fácil considerando las resistencias de los mismos docentes -en este caso académicos- frente a la evaluación de su desempeño o rendimiento en sus distintas modalidades: autoevaluación, evaluación por pares, estudiantes a través de la aplicación de las encuestas en el aula, entre otros. 


\section{Acerca de la Universidad de Santiago de Chile y la Facultad Tecnológica}

Los antecedentes más actualizados de la USA$\mathrm{CH}$, los podemos encontrar en el Plan Estratégico Institucional 2011-2015 (2012). En este Plan "se pudo se pudo arribar a fundamentos consensuados que han permitido reafirmar nuestra identidad y construcción institucional. Este proceso comenzó a mediados del año 2009 y concluyó a finales del 2011" (p. 2). De acuerdo con este plan, la Universidad de Santiago de Chile, es una institución de educación pública superior, estatal, con goce de autonomía y heredera de una centenaria tradición al progreso del país. Su propósito es crear, preservar, difundir y aplicar el conocimiento para el bienestar de la sociedad a través de la docencia, investigación y extensión. "Su misión es generar las condiciones y oportunidades que posibiliten a las generaciones futuras hacerse cargo del avance del conocimiento, su transferencia y retroalimentación para el crecimiento y desarrollo del país en una sociedad global" (p. 5). En el cumplimiento de esta misión, la Universidad procura alcanzar los más altos estándares de calidad, innovación y pertinencia en un ambiente de mutuo respeto entre las personas, libertad de pensamiento, diversidad e inclusión social; generando espacios de discusión y proposición en un diálogo multidisciplinario y pluralista. Lo anterior se traduce en la formación de personas, enriqueciendo su capital cultural y su compromiso social, impulsando la apertura de sus estudiantes al conocimiento y la comprensión de la interrelación que tienen con el entorno nacional e internacional (p. 5).

Por otra parte y respecto de la Visión de la organización, el Plan Estratégico antes señalado plantea que la Universidad de Santiago de Chile es "protagonista permanente del progreso del país, se proyecta como una comunidad universi- taria inclusiva, que crece y se desarrolla responsablemente en áreas claves y pertinentes, que aporten efectivamente a la ampliación del conocimiento y la generación de bienes públicos, necesarios para la construcción de una sociedad democrática y equitativa" (p.5).

La Universidad de Santiago de Chile se organiza en siete Facultades, una de ellas es la Facultad Tecnológica. Esta unidad académica mayor tiene su origen en 1969, cuando el Consejo Superior de la Universidad Técnica del Estado crea carreras tecnológicas destinadas a formar profesionales de mando medio o táctico, cuya demanda era apremiante en el sector productivo -tanto industrial como de servicios- de aquellos años. Es en el mes de junio de 1994 de la otrora Escuela Tecnológica nace la Facultad Tecnológica tal como la conocemos hoy en día. Integran su estructura organizativa cinco unidades: el Departamento de Ciencia y Tecnología de los Alimentos (DECYTAL), Departamento de Tecnologías Generales (DTG), Departamento de Tecnologías Industriales (DTI), Departamento de Gestión Agraria (DGA) y el Programa de Publicidad.

De acuerdo con el Plan Estratégico 2007-2011 (2007) está en su visión "ser reconocida nacional e internacionalmente por su liderazgo en la generación, desarrollo, aplicación, innovación y transmisión del conocimiento tecnológico, identificada con los valores, raíces y esencia de la Universidad de Santiago de Chile"(p.7). Por otra parte, al plantear su misión, esta "se identifica con el desarrollo, aplicación e innovación y transmisión del conocimiento tecnológico a través de la docencia, investigación y transferencia tecnológica contribuyendo a satisfacer las necesidades de la sociedad en el ámbito de su quehacer" (p. 7).

La oferta formativa actual de la Facultad está compuesta por las siguientes carreras: Inge- 
niería de Alimentos, Ingeniería en Agronegocios, Publicidad, el programa de Licenciatura en Organización y Gestión Tecnológica y la carrera de Tecnólogo en ocho especialidades: Administración de Personal, Alimentos, Automatización Industrial, Control Industrial, Construcciones, Diseño Industrial, Mantenimiento Industrial y Telecomunicaciones. Cabe mencionar que todas sus carreras con ingreso vía prueba de selección universitaria (PSU) se encuentran acreditadas, con un promedio de 4,9 años.

\section{Con relación al Reglamento de Evaluación y Calificación del Desempeño Académico}

Concebido en un marco en el cual para la Universidad el aseguramiento de la calidad constituye un compromiso permanente, la necesidad de aplicar procesos de evaluación y calificación del desempeño destinados a apreciar y medir la calidad y productividad de sus académicos y que para lograr este propósito se requiere contar con un instrumento que asegure la objetividad e imparcialidad y establezca un procedimiento de carácter general, que se realice en forma regular y la necesidad de avanzar hacia la confección de los respectivos Manuales de Evaluación y Calificación del Desempeño Académico y luego de una cantidad importante de gestiones ante la Contraloría General de la República, finalmente con fecha 03 de agosto del año 2009 se aprueba el Reglamento de Evaluación y Calificación del Desempeño Académico' para esta casa de estudios.

Esta normativa tiene por objeto evaluar y calificar el trabajo realizado por cada uno de los

1. Universidad de Santiago de Chile (2009). Resolución exenta Aprueba Reglamento de Evaluación y calificación del Desempeño Académico. Santiago de Chile. académicos de la Universidad; constituir un incentivo permanente para el perfeccionamiento de las actividades que sirvan para promover y desarrollar su cuerpo académico y contribuir, en suma, a elevar los niveles de calidad y productividad de la Universidad.

Entre los principales conceptos que se definen desde el inicio del Reglamento de Evaluación y Calificación del Desempeño Académico, se destaca el de Evaluación de Desempeño; en esta ocasión la normativa lo define como el "procedimiento que mide tanto el grado de cumplimiento, como la calidad de las actividades académicas debidamente comprobadas de un académico, durante el período sujeto a evaluación y que culmina con la calificación respectiva. Para este propósito se compara la actividad realizada con los compromisos acordados previamente con el Director de Departamento o Unidad Equivalente" (p. 2).

Las actividades académicas susceptibles de evaluación son las siguientes:

\section{Docencia}

Se considerarán actividades docentes, entre otras: la planificación, diseño y evaluación de planes y programas de pre, postgrado y postítulo, docencia en aula o a distancia, dirección de trabajos conducentes a títulos profesionales o a grados académicos, gestión y administración de carreras, publicaciones y comunicaciones docentes, proyectos de investigación y/o desarrollo docente, perfeccionamiento o actualización en docencia.

\section{Investigación}

Se considerarán actividades de investigación, entre otras, las siguientes: participación, ya sea como investigador principal, investigador asociado o consultor, en proyectos de investigación; planificación, diseño e implementación de proyectos de investigación; gestión administrativa y 
desarrollo de proyectos de investigación; organización de encuentros científicos; elaboración de productos de los proyectos de investigación (libros, capítulos de libros, artículos en revistas, patentes, entre otros); perfeccionamiento en investigación; dirección de tesis de postgrado en investigación; participación en programas de postgrado.

\section{Asistencia Técnica}

Se considerarán actividades de asistencia técnica, entre otras, las siguientes: formulación de proyectos de asistencia técnica como responsable o colaborador, gestión y desarrollo de proyectos de asistencia técnica; formulación de proyectos de capacitación en calidad de responsable o colaborador; gestión y desarrollo de proyectos de capacitación en calidad de responsable o docente.

\section{Extensión}

Se considerarán actividades de extensión, entre otras, las siguientes: conferencias, charlas, participación en seminarios, mesas redondas, programas de radio y televisión, artículos en prensa, artículos de divulgación científica y técnica.

Otras actividades académico administrativas no consideradas en las áreas anteriores, tales como Consejeros de Departamentos, Consejeros de Facultad, Consejeros Académicos de la Universidad, cargos de representación en organismos nacionales o internacionales, en consejos y comités editoriales de revistas profesionales y científicas (p. 2-3).

Cabe mencionar que esta normativa señala explícitamente en su título VIII que el procedimiento de evaluación y calificación se regirá, en lo sustantivo, por lo dispuesto en los manuales que para ese efecto hayan sido aprobados por los respectivos Consejos de Facultad y validados por la Comisión Superior (p.7).

\section{Desafíos para la Facultad Tecnológica de la Universidad de Santiago con relación a la Evaluación y Calificación del Desempeño Académico}

La Facultad Tecnológica de la USACH junto con haber realizado un plan piloto, aplicar el Reglamento en su primera fase durante el año 2011, ha concluido la confección de su Manual de Desempeño. Manual que se encuentra hoy en análisis y homologación por parte de la Comisión Superior de Evaluación y Calificación. A esta fecha, los principales desafíos están muy vinculados a las tensiones y dificultades que se aprecian en lo que ha sido y seguirá siendo su aplicación práctica. Entre los principales podemos mencionar:

1. Falta de consenso en los distintos niveles operacionales respecto de las unidades de medida asociadas a los roles y áreas que van a ser evaluadas y calificadas. Si bien es cierto -el reglamento lo señala- se entiende que serán medidos en los ámbitos propios del quehacer de la universidad, tales como Docencia, Investigación, Asistencia Técnica y Extensión, no existe total claridad de los diferentes roles que asume un académico en cada dimensión y cómo se valorará esa particular contribución.

2. Necesidad de relacionar los roles y unidades de medida a la jerarquía de cada Académico. En estos momentos, a nivel de Facultad, no se aprecia claramente la diferenciación de actividades por área según si el docente es de Jerarquía Titular, Asociado, Asistente o Instructor. Este tema no es menor dado que la base de la evaluación es el Convenio de Desempeño que cada académico firma con 
su Director de Departamento, perfectamente podría darse el caso que un académico Titular realice actividades de una Jerarquía menor bastando el acuerdo con su jefe directo.

3. Necesidad de precisar y diferenciar los criterios de cada categoría. En estos momentos las categorías son: sobresaliente, bueno, aceptable, condicional e insuficiente. Estas categorías a su vez se asocian a notas que van entre 0 a 4 . El problema o la dificultad es que en algunos aspectos tales como Docencia en aula, Guía de Seminario o Trabajos de Titulación y Tesis de Grado, Elaboración de Textos, Perfeccionamiento, en fin; no hay claridad ni diferenciación nítida en los criterios por ejemplo, para ser evaluado en la categoría de sobresaliente o de bueno.

4. Articulación de los distintos actores involucrados en el proceso en los roles de evaluadores y calificadores. Según el reglamento respectivo participarán en el proceso los siguientes órganos: Comisión de Departamento, Comisión de Facultad, Consejo de Facultad y la denominada Comisión Superior de Calificación y Desempeño Académico. Además de ser variados los órganos que participarán en la evaluación y calificación se teme o preocupa que el sistema se ultra burocratice arrojando más dificultades y problemas que las ventajas y conveniencias para las cuales fue instaurado.

5. Reales efectos de la evaluación y calificación del desempeño académico. Si bien es cierto el reglamento se refiere a consecuencias tales como promoción en la carrera académica, estímulos de desempeño, programas de capacitación y desarrollo, y desvinculación de la universidad, existe en la comunidad la "sensación" que puede ser utilizada preferentemente con fines "punitivos" muy contrario al espíritu de las modernas políticas de gestión recursos humanos en este sentido.

\section{CONCLUSIONES}

Ya lo ha sostenido Rodríguez (2008) al señalar que las relaciones entre las personas y la organización en donde hace su contribución y/o aporte es altamente relevante impactando tanto en la rentabilidad de la empresa como el bienestar en el trabajo. Textualmente ha expresado:

Cada trabajador dedica parte importante de su vida a la organización de la que es miembro activo, por lo que su mayor o menor bienestar laboral en ella va a redundar en forma determinante en su calidad de vida. A esto se agrega que hoy en día se ha puesto de relieve la incidencia que tiene el factor humano en la calidad y productividad del trabajo realizado en las organizaciones. La forma de establecer un vínculo satisfactorio es, por consiguiente, algo que ha de ser buscado y conseguido en las organizaciones modernas (p.135).

Continuando con esta línea argumentativa, podemos decir que la Evaluación del Desempeño también debiera aspirar a relevar la importancia estratégica del factor humano en la organización y cómo este influye o impacta en la calidad y productividad del trabajo que se realiza. Según los ya citados Jorge Catalán y Mauricio González -considerando el caso de una Institución educativa como la USACH- de lo que se trata es "de hacer mensurable u observable lo que los docentes deben saber y saben hacer en el ejercicio de su profesión, de modo que la generación de estos estándares obedece al propósito de contribuir a la calidad docente y, por ende, de la educación, como también a favorecer un reconocimiento social acorde con el status profesional del profesor" (p. 5).

La Universidad de Santiago de Chile en el marco de procesos de aseguramiento de la calidad, necesidad de apreciar y valorar la contribución de sus docentes y el desarrollo de acciones sistemá- 
ticas de una gestión de recursos humanos, en el año 2009 aprobó el Reglamento de Evaluación y Calificación del Desempeño Académico. Esta normativa tiene por objeto evaluar y calificar el trabajo realizado por cada uno de sus académicos; constituir un incentivo permanente para el perfeccionamiento de las actividades que sirvan para promover y desarrollar su cuerpo académico y contribuir, en suma, a elevar los niveles de calidad y productividad de la Universidad. En este Reglamento de Evaluación y Calificación del Desempeño Académico, se define la Evaluación de Desempeño como el "procedimiento que mide tanto el grado de cumplimiento, como la calidad de las actividades académicas debidamente comprobadas de un académico, durante el período sujeto a evaluación y que culmina con la calificación respectiva". Para este propósito se compara la actividad realizada con los compromisos acordados previamente con el Director de Departamento o Unidad Equivalente. Cabe mencionar que la actividad realizada se desarrolla principalmente en los ámbitos de docencia, investigación, asistencia técnica y extensión.

Entre las principales dificultades y tensiones que se aprecian en lo que ha sido y seguirá siendo su aplicación práctica, podemos señalar: falta de consenso en los distintos niveles operacionales respecto de las unidades de medida asociadas a los roles y áreas que van a ser evaluadas y calificadas, la necesidad de relacionar los roles y unidades de medida a la jerarquía de cada académico o académica y los reales efectos de la evaluación y calificación del desempeño desde una perspectiva de gestión de personas. Esto implica también el desafío de relacionar este sistema con una efectiva gestión de recursos humanos en donde mucho más allá de los fines punitivos que parte de la comunidad visualiza, se promueva el perfeccionamiento continuo de los docentes y se les incentive y compense de acuerdo con sus méritos y productividad.
Como podemos apreciar, la implementación en la Universidad de Santiago de Chile de un sistema formal de Evaluación y Calificación del desempeño académico es un desafío mayor considerando los propósitos para los cuales fue diseñado, la cultura organizacional profundamente arraigada en la institución y lo que está más de fondo, esto es, el desarrollo permanente de su planta académica en un contexto de extrema competencia con otras instituciones educativas públicas y privadas del país y de la región.

\section{REFERENCIAS}

Catalán, J. y González, M. (2009): Actitud hacia la Evaluación del Desempeño Docente y su Relación con la Autoevaluación del Propio Desempeño, en Profesores Básicos de Copiapó, La Serena y Coquimbo. Psykhe, 18, pp. 4-5.

Dolan, S., Valle, R., Jackson, S. y Schuler, R. (2007). La gestión de los recursos humanos. Madrid, España: Mc Graw Hill.

Chiavenato, I. (1988). Administración de Recursos Humanos. México: Mc Graw Hill.

Fuchs, C. (1997). Sistema de Evaluación y Mejoramiento de Desempeño. Revista El Diario, pp.2.

Gan, F. y Triginé, J. (2006). Manual de Instrumentos de gestión y desarrollo de las personas en las organizaciones. España: Ediciones Díaz de Santos.

Páez, C. (2001): Evaluación del Desempeño. Revista Inter Forum, pp. 2-3.

Rodríguez, J. (2003): La evaluación del desempeño: Aproximación desde una perspectiva de género. Ciencia y Sociedad, 28, pp. 188-189.

Rodríguez, R. (2006): Evaluación de desempeño en 360 todos opinamos, todos nos beneficiamos. Psicología Científica.com, pp. 1-2.

Rodríguez, M. (2008). Gestión organizacional - elementos para su estudio. Chile: Universidad Católica de Chile.

Rueda, M. y Torquemada, A. (2008): Las concepciones sobre evaluación de profesores y estudiantes: sus repercusiones en la evaluación del desempeño docente. Reencuentro, 53, pp. 98-99. 
RNA Revista Nacional de Administración

Universidad de Santiago de Chile (2012). Plan Estratégico Institucional 2011 - 2015. pp. 5. Recuperado el 25-052012 de: http://www.pei.usach.cl/index/documentos

Universidad de Santiago de Chile (2009). Resolución exenta Aprueba Reglamento de Evaluación y calificación del Desempeño Académico. Santiago de Chile. pp. 2-3, 7.

Universidad de Santiago de Chile (2007). Plan Estratégico Facultad Tecnológica 2007 - 2011. pp. 7. Recuperado el 25-05-2012 de: http://www.factec.usach.cl/arch/doc/ documentos/PlanEstrategico.pdf

Recibido: 16-12-2011

Aceptado: 08-02-2012 